\title{
Defensas antioxidantes en el fluido celómico del erizo negro Echinometra lucunter (Linnaeus, 1758) estimulado con inóculos bacterianos
}

\section{Antioxidant defenses in the coelomic fluid of Echinometra lucunter (Linnaeus, 1758) stimulated with bacterial inoculums}

\author{
Edgar Zapata-Vívenes ${ }^{1,2 *}$ y Gabriela Aparicio ${ }^{3}$
}

\begin{abstract}
RESUMEN
La fagocitosis es una respuesta celular de primera línea mediada por células especializadas denominadas celomocitos-amebocitos. Este proceso permite englobar partículas extrañas o microorganismos, los cuales son eliminados a través de la generación de especies reactivas del oxígeno (ERO). Con la finalidad de evaluar el sistema de defensa antioxidante en el fluido celómico (FC) del erizo de mar negro E. lucunter bajo una alta actividad fagocítica (AF), se procedió a inocular vía membrana peristomial tres cepas bacterianas por separado: $E$. coli, $V$. parahaemolyticus y M. lysodeikticus. A las $16 \mathrm{~h}$ posinyección, se determinaron la capacidad fagocítica (CF), actividad de catalasa (CAT) y superóxido dismutasa (SOD), niveles de lipoperoxidación (LPO), grupos sulfhidrilos (-SH) y proteínas. Adicionalmente, fue estimado el tiempo de enderezamiento de cada individuo. La CF, CAT y proteínas mostraron incrementos en los organismos inyectados con inoculados bacterianos. Los niveles de LPO, SOD y -SH no mostraron variaciones entre los organismos experimentales. El tiempo de enderezamiento presentó ligeras variaciones en los organismos estimulados con bacterias; revelando a su vez un bajo porcentaje de pérdida y reducción de movimiento de sus espinas y pies ambulacrales. La AF y las proteínas en FC de E. lucunter muestran la efectividad del sistema inmune en presencia de estimulantes microbianos. Los resultados indican que CAT juega un papel preponderante en el FC para evitar cambios en el estatus antioxidante, asociados al estallido respiratorio frente a actividades fagocitarias elevadas. Las respuestas antioxidantes de E. lucunter inmuno-estimulado por bacterias pueden garantizar su sobrevivencia en el hábitat natural.
\end{abstract}

Palabras clave: Catalasa, peroxidación lípidos, estrés oxidativo, E. coli, tioles

1 Departamento de Biología, Escuela de Ciencias, Núcleo de Sucre, Universidad de Oriente, Cumaná, Venezuela. ezapata@udo.edu.ve*, ezapatavivenes@gmail.com

2 Escuela de Acuicultura y Pesca, Facultad de Ciencias Veterinarias, Universidad Técnica de Manabí, Bahía de Caráquez, Manabi, Ecuador.

3 Departamento de Bioanálisis, Escuela de Ciencias, Núcleo de Sucre, Universidad de Oriente, Cumaná, Venezuela. 


\section{ABSTRACT}

Phagocytosis is a first-line cellular response mediated by specialized cells denominated coelomocytes- amoebocytes. This process allows for the inclusion of extraneous particles or microorganisms, which are eliminated by the production of reactive oxygen species (ROS). In order to evaluate the antioxidant defense system in the coelomic fluid (CF) of E. lucunter under elevated phagocytic activity (PA), three bacterial strains were inoculated separately, via peristomial membrane: E. coli, V. parahaemolyticus and M. lysodeikticus. The phagocytic capacity (PC), catalase (CAT) and superoxide dismutase (SOD) activities, levels of lipoperoxidation (LPO), sulphydryl groups (-SH) and proteins were determined at $16 \mathrm{~h}$ post-injection. In addition, the time of righting response of each individual was noted. The PA, CAT, and proteins showed increments in the organisms inoculated with bacteria. The LPO, SOD, and -SH levels showed no variations among the experimental organisms. The time of righting response showed slight variations in the bacteria-stimulated organisms, as well as a low percentage of lost and reduced movements of their spines and tube feet. The PA and proteins in CF of E. lucunter show the effectiveness of the immune system in the presence of microbial stimulants. Results indicate that CAT plays a preponderant role in the CF to avoid changes in the antioxidant status, associated with the respiratory burst during elevated phagocytic activities. Antioxidant responses of $E$. lucunter immunostimulated by bacteria may guarantee their survival in their natural habitat.

Keywords: Catalase, lipid peroxidation, oxidative stress, E. coli, thiols

\section{INTRODUCCIÓN}

El erizo de mar Echinometra lucunter es una especie de relativa abundancia en la costa nororiental de Venezuela (Reyes-Luján et al. 2015), que presenta potencialidades para el maricultivo. Su expectativa de cultivo posiblemente puede ser limitada por enfermedades provocadas por microorganismos patógenos, en especial bacterias (Wang et al. 2012a). Tales microorganismos pueden ocasionar lesiones en las paredes de la testa de los erizos, pérdida de la pigmentación del epitelio, desprendimiento y crecimiento irregular en las puntas de las espinas, necrosis de tejidos musculares, dificultad en la alimentación y locomoción (Bauer \& Young 2000; Wang et al. 2012b). Los géneros bacterianos, frecuentemente aislados de áreas corporales de erizos de mar lesionados son Vibrio, Aeromonas, Flavobacterium, Clostridium y Pseudomonas (Wang et al. 2012b).

Los individuos más propensos a enfermarse son los que presentan signos de estrés asociado a una deficiencia inmunológica. Los erizos de mar, así como otros invertebrados, no poseen un sistema inmune adaptativo, sino que dependen totalmente de las respuestas celulares y humorales innatas para la protección contra patógenos (Antón \& Salazar, 2009; Branco et al. 2014). La inmunidad en los erizos de mar está a cargo de diversos tipos de células denominadas celomocitos (amebocitos, granulocitos traslucidos, 
esférulas rojas y células vibrátiles) y sustancias bacteriolíticas contenidas en su fluido celómico (FC) (Matranga et al. 2005; Pinsino \& Matranga, 2015).

El sistema inmunológico de los erizos de mar puede ser inducido mediante invasiones bacterianas lo que conlleva a incrementar la actividad de diversos agentes antimicrobianos, el número de celomocitos y la capacidad fagocítica. En este último evento se produce un aumento en la producción de especies reactivas del oxígeno (ERO), con capacidad bactericida, tales como el anión superóxido $\left({ }^{\bullet} \mathrm{O}_{2}\right)$, y este, a su vez, es convertido a peróxido de hidrógeno $\left(\mathrm{H}_{2} \mathrm{O}_{2}\right)(\mathrm{Cou}-$ ter et al. 1999; Mydlarz et al. 2006). Las ERO pueden dañar también a las células del hospedero, siendo controladas por las enzimas del sistema de defensa antioxidante tales como superóxido dismutasa (SOD) y catalasa (CAT), para evitar afectos oxidativos sobre proteínas, ADN y lípidos (Halliwell \& Gutteridge, 2015; Chihuailaf et al. 2002). Otras sustancias con potencialidades antioxidantes son las moléculas que contienen grupos tioles o sulfhidrilos (-SH); estos grupos funcionales desempeñan un papel importante en los sistemas biológicos (Baba \& Bhatnagar, 2018).

Se ha demostrado la efectividad de las respuestas inmunológicas en erizos de mar estimuladas por distintos antígenos de origen bacteriano (Dhei1ly, 2010; Dheilly et al. 2012; Bastidas,
2017). Sin embargo, se sabe muy poco acerca de cómo modulan sus defensas antioxidantes los erizos de mar en presencia de una alta actividad fagocítica. En tal sentido, esta investigación evaluó la capacidad del sistema de defensa antioxidante en E. lucunter al ser estimulado por distintas cepas bacterianas inoculadas experimentalmente.

\section{MATERIALES Y MÉTODOS}

Organismos: los ejemplares de E. lucunter con talla $>6 \mathrm{~cm}$ de diámetro fueron colectados de manera manual, por buceo libre, a profundidades de 1 a $5 \mathrm{~m}$ en la ensenada de Turpialito $\left(10^{\circ} 26^{\prime} 56^{\prime \prime} \mathrm{N}, 64^{\circ} 02^{\prime} 00^{\prime}\right.$ ' O) (Fig. 1).

Esta localidad se encuentra situada a $3 \mathrm{~km}$ al este de la ciudad de Cumaná, en la costa sur del golfo de Cariaco, Venezuela.

\section{Manutención en el laborato-} rio: los ejemplares fueron transportados en envases isotérmicos hasta el laboratorio y se colocaron en acuarios de $40 \mathrm{~cm}^{3}$, a razón de un organismo por cada 5 litros de agua de mar filtrada, con suficiente aireación, temperatura promedio del agua $25 \pm 1{ }^{\circ} \mathrm{C} ; \mathrm{pH}$ 7.9-8.1 y salinidad de 36 UPS, durante una semana. Los organismos fueron alimentados diariamente con una mezcla de macroalgas deshidratadas de los géneros Sargassum, Padina y Ulva. El recambio de agua de mar de los acuarios fue realizado cada dos días. 


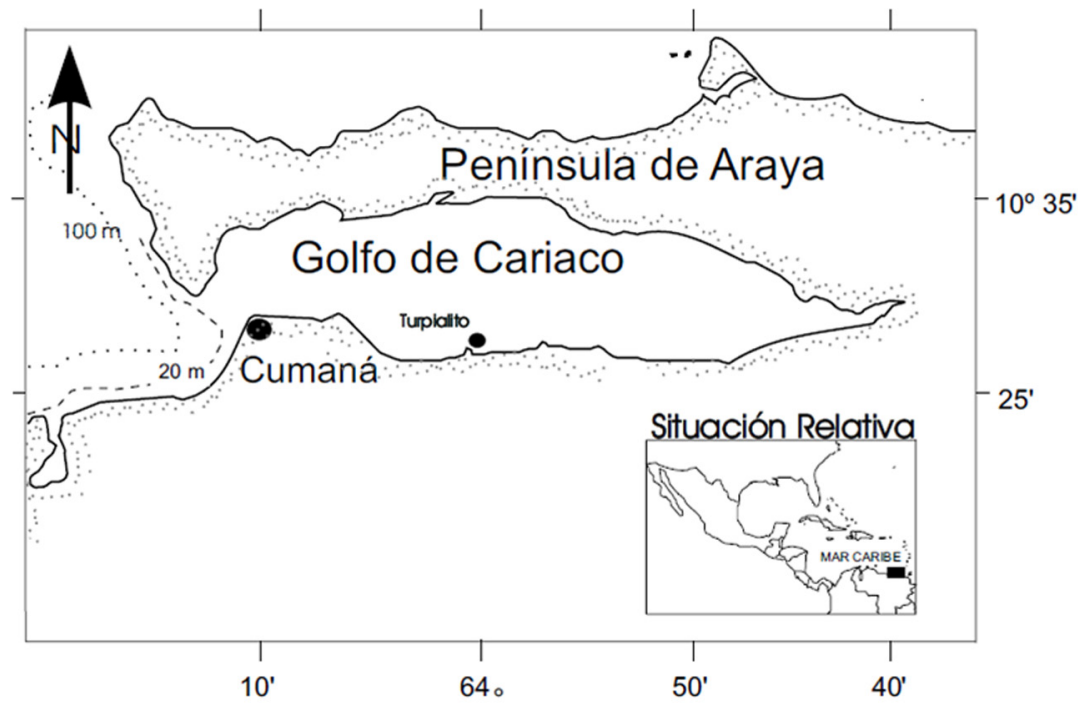

Fig. 1. Ubicación geográfica de la ensenada de Turpialito, Golfo de Cariaco, Venezuela

Fig. 1. Geographic location of the Turpialito cove, Gulf of Cariaco, Venezuela

Ensayos de inoculación: cuatro grupos de animales por separado, constituidos de 10 organismos cada uno, se les inyectaron, cuidadosamente dentro de la cavidad peristomial (entrada aboral), concentraciones conocidas de las siguientes bacterias: $E s-$ cherichia coli (ATCC 25522), Vibrio parahaemolyticus (cepa HUAPA) y Microccocus lysodeikticus (SIGMA N. $\left.{ }^{\circ} 4698\right)$. Al primer y segundo grupo, se les inoculó $100 \mu$ l de una solución contentiva de $\sim 1 \times 10^{8}$ células $/ \mathrm{ml}$ de E. coli y $V$. parahaemolyticus, disueltas en agua de mar estéril. Al tercer grupo de animales se le inyectó 100 $\mu 1$ de una solución de M. lysodeikticus $\left(\sim 1 \times 10^{8}\right)$. Al grupo cuarto grupo de organismos se le introdujo el mismo volumen de agua de mar micro-filtrada y estéril (considerada solución salina). Una vez inyectados cada uno de los organismos fueron devueltos a sus respectivos acuarios.

Además, se utilizó como grupo control (no inyectados) a organismos provenientes del campo, previamente aclimatados y no estresados por punción. La obtención del FC se realizó a las 16 horas posinyección bacterial. Los experimentos fueron realizados por duplicados.

\section{Obtención de fluido celómico} (FC): para ello, se insertó una aguja hipodérmica $25 \mathrm{G} 5 / 8^{\prime \prime}$, contentiva de $200 \mu 1$ de agua de mar estéril y EDTA a $4 \mathrm{mmol} / 1$. Se obtuvo un máximo de $1 \mathrm{ml}$ por ejemplar. El FC fue coloca- 
do en un tubo eppendorf en hielo y se adicionó $20 \mathrm{mmol} / 1$ Tris $0.5 \mathrm{~mol} / \mathrm{l} \mathrm{de}$ $\mathrm{NaCl}$ y $30 \mathrm{mmol} / \mathrm{l}$ de ácido etilendiami-tetracético (ISO-EDTA, pH 7.50) como solución anticoagulante (Matranga et al. 2006).

Actividad fagocítica: fue determinada mediante la reducción del azul de tetrazolium (NBT) a formazán (Cooper et al. 1995), utilizando como antígeno un extracto no viable de la levadura Saccharomyces cerevisiae (zymozan). Se mezclaron $100 \mu \mathrm{l}$ del extracto de zymozan $(10 \mu \mathrm{g} / \mathrm{ml})$ con $100 \mu \mathrm{l}$ de suspensión de celomocitos $\left(\sim 6 \mathrm{x} 10^{5} \mathrm{cel} /\right.$ $\mathrm{ml})$, y luego se incubó por 15 minutos. Se le agregó $100 \mu \mathrm{l}$ de una solución de NBT $(2 \mathrm{mg} / \mathrm{ml})$ en buffer fosfato salino (BFS), se mezcló y se incubó a $16^{\circ} \mathrm{C}$ bajo oscuridad, por 24 horas. Para extraer el formazán (producto de la reducción del NBT), se agregaron $200 \mathrm{ml}$ de hexadecyltrimetil amonio bromuro $0.1 \%$ como agente antimicrobiano y $400 \mathrm{ml} \mathrm{de} \mathrm{buffer} \mathrm{fosfato} 100 \mathrm{mmol} / \mathrm{l}$ $\mathrm{pH}$ 6.2. Seguido de un período de incubación de 3 horas a $37^{\circ} \mathrm{C}$ y una centrifugación posterior a $2000 \mathrm{~g}$ por 15 min, se procedió a medir la absorbancia a $510 \mathrm{~nm}$. Estas lecturas fueron comparadas con una curva patrón de formazán realizado con bromuro 3-(4,5-dimetilthiazol-2-yl)-2,5-difeniltetrazolium (Sigma-Aldrich Cat. M2128). Como control se cuantificó el formazán en celomocitos incubados con NBT sin antígeno. Los resultados se expresaron en $\mu \mathrm{g}$ de formazán $/ \mathrm{ml}$.
Peroxidación de lípidos: fue estimada por el método de análisis de sustancias reactivas del ácido tiobarbitúrico (TBARS) al seguir el protocolo estandarizado propuesto por Ohkawa et al. (1978). La concentración de TBARS se calculó usando un coeficiente de extinción de $1.56 \times 10^{5} 1 \mathrm{~cm} /$ mmol. Los resultados son expresados en milimoles de TBARS por miligramos de proteínas.

Proteínas totales: se determinó por el método de Biuret. Se tomaron $100 \mu \mathrm{lde}$ FC y $1 \mathrm{ml}$ del reactivo de Biuret, se agitaron suavemente los tubos y se dejaron reposar por 20 minutos; luego se midió la absorbancia a $540 \mathrm{~nm}$ (Robinson \& Hogden, 1940). Se contrastó con una curva patrón realizada con albúmina de suero de bovino $(1 \mathrm{mg} / \mathrm{ml})$.

Superóxido dismutasa: se determinó mediante la utilización de un Kit Bioxytech SOD-525 (SIGMA), el cual se fundamenta en la medición del incremento de la tasa de auto-oxidación del 5,5,6a,11b-tetrahidro-3,9,10-trihidrobenzo[c]fluoreno (TTF), mediado por la enzima SOD en solución acuosa alcalina, para producir un cromógeno con un máximo de absorbancia a 525 nm (Nebot et al. 1993).

Catalasa: la actividad de la enzima CAT se determinó por el descenso de la concentración del peróxido de hidrógeno $\left(\mathrm{H}_{2} \mathrm{O}_{2}\right)$ a una longitud de onda de $240 \mathrm{~nm}$ (Coeficiente de Extinción $=401 \mathrm{~cm} / \mathrm{mmol})$ (Aebi, 1984). 
Análisis enzimáticos: la actividad de las enzimas antioxidantes fue determinada en un espectrofotómetro Perkin-Elmer UV/VIS Lambda 2S, bajo condiciones de temperatura controlada a $25 \pm 0.5^{\circ} \mathrm{C}$. Los ensayos enzimáticos se realizaron por triplicado en un volumen final de $1 \mathrm{ml}$. Las estimaciones de las actividades enzimáticas se realizaron con en el programa UV Winlab Lambda 25 bajo ambiente Windows.

\section{Grupos sulfhidrilos totales} (-SH): se determinó por el método de Ellman (1959). En la cubeta experimental se añadieron $50 \mu \mathrm{l}$ de FC, 500 $\mu 1$ de DTNB, $250 \mu 1$ de agua destilada, $200 \mu 1$ de buffer Tris y $50 \mu 1$ de la muestra se incubó por 10 minutos a temperatura ambiente. Luego se midió la absorbancia a $412 \mathrm{~nm}$, contra un blanco constituido por todos los reactivos a excepción del extracto. Se usó al GSH a $3 \mathrm{mmol} / 1$ como estándar.

Salud de los erizos de mar: se determinó mediante el tiempo de recuperación de una posición invertida. Los erizos fueron colocados en posición invertida (boca arriba) en el fondo del acuario. Se estimó el tiempo que tardó el organismo en voltearse para alcanzar su posición original (Taylor et al. 2014). Adicionalmente, en organismos sensibilizados se monitoreó diariamente durante una semana algunos síntomas, tales como cambios de coloración, pérdidas de espinas, comportamientos anormales.

\section{Análisis estadísticos}

Los resultados de los tratamientos en los grupos estudiados se compararon con los controles a través de un análisis de varianza de una vía (ANOVA). La normalidad de los datos y la homogeneidad de las varianzas fueron verificadas; solo la actividad de CAT y SOD fueron transformadas en $\log _{10}$ para normalizar su distribución. Para el contraste múltiple de rango se utilizó la prueba de Bonferroni. Los análisis estadísticos fueron realizados bajo el programa estadístico SPSS versión 11.5 (Sokal \& Rohlf, 1981).

\section{RESULTADOS}

La actividad fagocítica de los celomocitos incrementó significativamente en todos los erizos de mar inyectados (ANOVA, GL=64 Fs=7.14; $P<0.05)$. La mayor actividad de fagocitosis fue registrada en los erizos de mar inyectados con V. parahaemolyti$c u s$, seguidos por el grupo formado por los organismos inyectados con $M$. lysodeikticus y E. coli, inclusive los inyectados con solución salina (Fig. 2).

La actividad de CAT mostró incrementos significativos en los organismos inoculados con las cepas bacterianas $M$. lysodeikticus, V. parahaemolyticus, y $E$. coli con respecto al grupo no inyectado, no así en los organismos inyectados con solución salina (ANOVA, $\mathrm{GL}=49$; $\mathrm{Fs}=8.23 P<0.05)($ Fig. 3). La enzima 


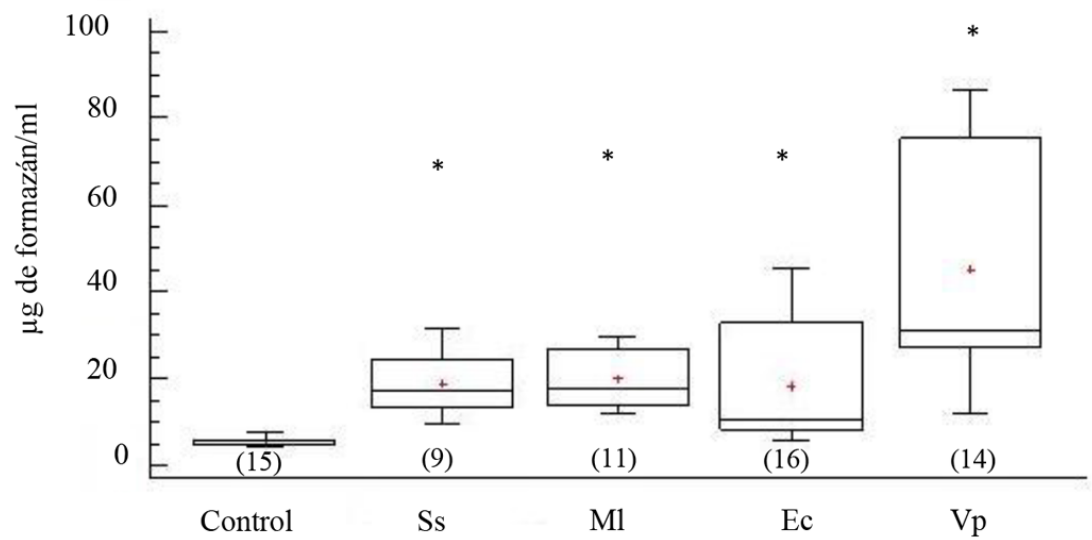

Fig. 2. Niveles de formazán en fluido celómico del erizo de mar Echinometra lucunter inoculados con solución salina (Ss), M. lysodeikticus (Ml), E. coli (Ec) y $V$. parahaemolyticus (Vp). El asterisco muestra diferencias significativas $* P<0.05$. (N) indica en número de individuos analizados

Fig. 2. Formazan levels in coelomic fluid of Echinometra lucunter inoculated with saline solution (Ss), M. lysodeikticus (Ml), E. coli (Ec), and V. parahaemolyticus (Vp). (*) The asterisk shows significant differences $* P<0.05$. (N) Number of individuals analyzed

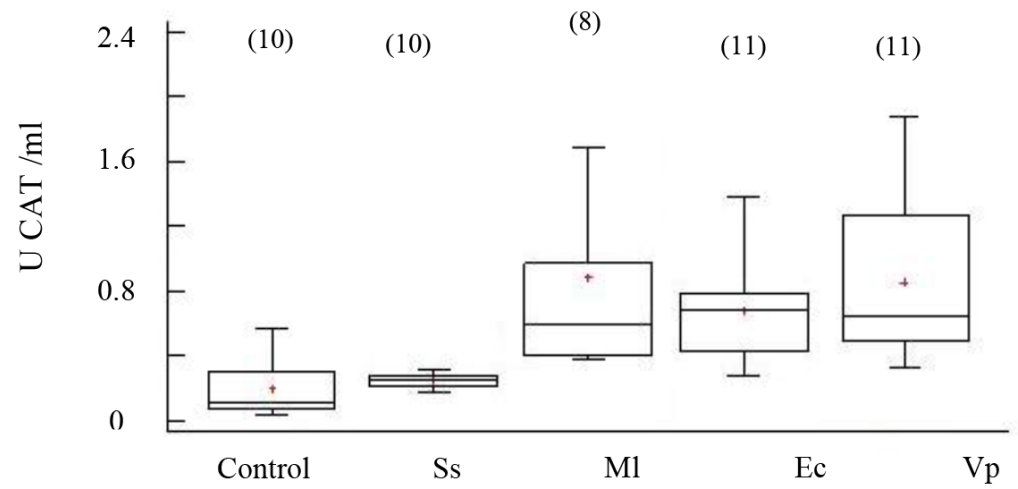

Fig. 3. Actividad de catalasa (CAT) en fluido celómico del erizo de mar Echinometra lucunter inoculados con solución salina (Ss), M. lysodeikticus (Ml), E. coli (Ec) y $V$. parahaemolyticus (Vp). (*) El asterisco muestra diferencias significativas $* P<0.05$. (N) indica en número de individuos analizados

Fig. 3. Catalase activity (CAT) in coelomic fluid of Echinometra lucunter inoculated with saline solution (Ss), M. lysodeikticus (Ml), E. coli (Ec), and $V$. parahaemolyticus $\left.(\mathrm{Vp}) .{ }^{*}\right)$ The asterisk shows significant differences $* P<0.05$. (N) Number of individuals analyzed 
superóxido dismutasa (SOD) no evidenció cambios significativos en su actividad entre los organismos inoculados con cepas bacterianas y los no inoculados (Fig. 4); aunque sus mayores valores se registraron en los organismos inoculados con $V$. parahaemolyticus (ANOVA, GL=50 Fs=1.25; $P>0.05$ ).

Los niveles de grupos -SH en el FC de los organismos, aparentemente sanos e inoculados con las distintas bacterias, no mostraron diferencias estadísticamente significativas entre grupos (ANOVA, $\mathrm{GL}=70 ; \mathrm{Fs}=0.77$ $P>0.05$ ) (Fig. 5). Tampoco, los niveles de peroxidación lipídica, evidenciaron diferencias significativas entre los organismos experimentales (ANOVA, $\mathrm{GL}=67 ; \mathrm{Fs}=0.54 P>0.05$ ) (Fig. 6).

Los niveles de proteínas totales por mililitro de FC indican diferencias significativas (ANOVA, GL=68; Fs $=6.63 P<0.05$ ), en especial en los organismos inyectados $V$. parahemolyticus (Fig. 7). El tiempo de recuperación de posición invertida permitió indicar la salud de los erizos de mar, al evidenciar los promedios en tiempo de enderezamiento en los organismos controles e inoculados en un rango entre 40 a $100 \mathrm{~s}$ (Fig. 8), sin mostrar diferencia estadística entre los tratamientos (ANOVA, GL=91; Fs=1.42 $P>0.05$ ).

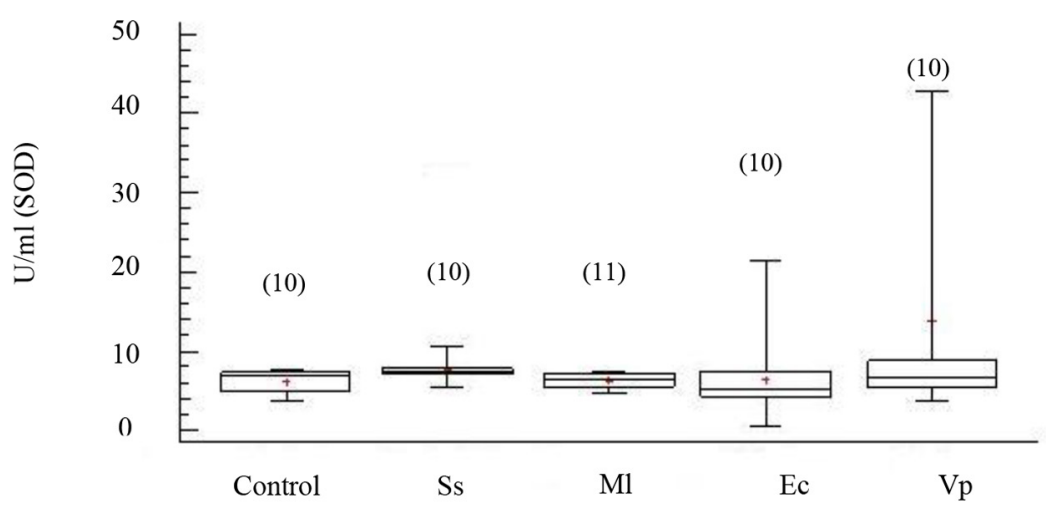

Fig. 4. Actividad de superóxido dismutasa (SOD) en fluido celómico del erizo de mar Echinometra lucunter inoculados con solución salina (Ss), M. lysodeikticus $(\mathrm{Ml})$, E. coli $(\mathrm{Ec})$ y V.parahaemolyticus $(\mathrm{Vp})$. (N) indica en número de individuos analizados

Fig. 4. Superoxide dismutase (SOD) activity in coelomic fluid of Echinometra lucunter inoculated with saline solution (Ss), M. lysodeikticus (Ml), E. coli (Ec), and $V$. parahaemolyticus $(\mathrm{Vp})$. (N) Number of individuals analyzed 


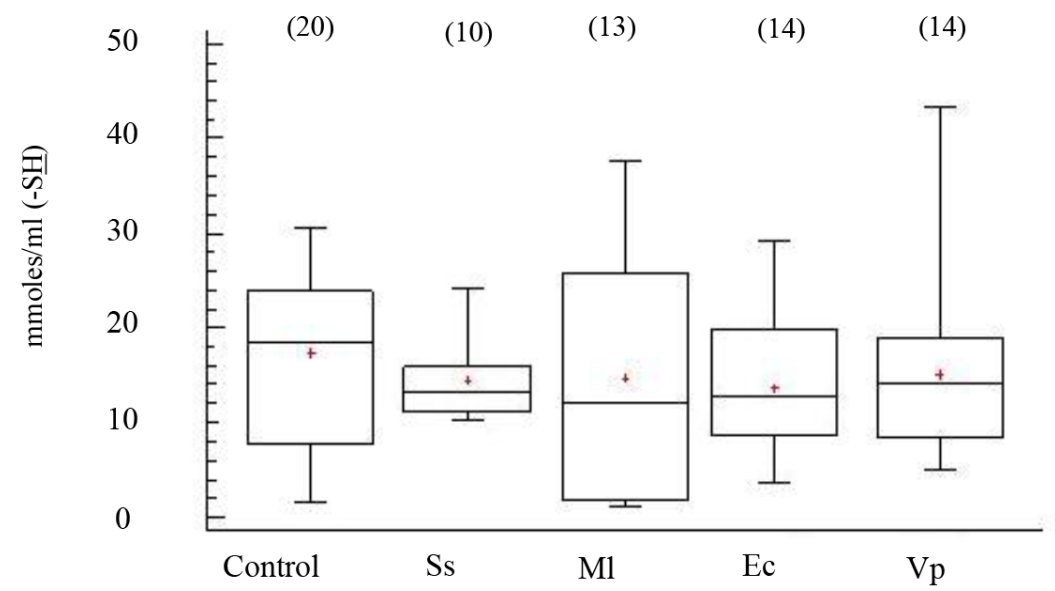

Fig. 5. Niveles de grupos sulfhidrilos totales (-SH) en fluido celómico del erizo de mar Echinometra lucunter inoculados con solución salina (Ss), $M$. lysodeikticus $(\mathrm{Ml})$, E. coli $(\mathrm{Ec})$ y $V$. parahaemolyticus $(\mathrm{Vp})$. (N) indica en número de individuos analizados

Fig. 5. Levels of sulphydryl (-SH) groups in coelomic fluid of Echinometra lucunter inoculated with saline solution (Ss), M. lysodeikticus (Ml), E. coli (Ec), and $V$. parahaemolyticus $(\mathrm{Vp})$. (N) Number of individuals analyzed

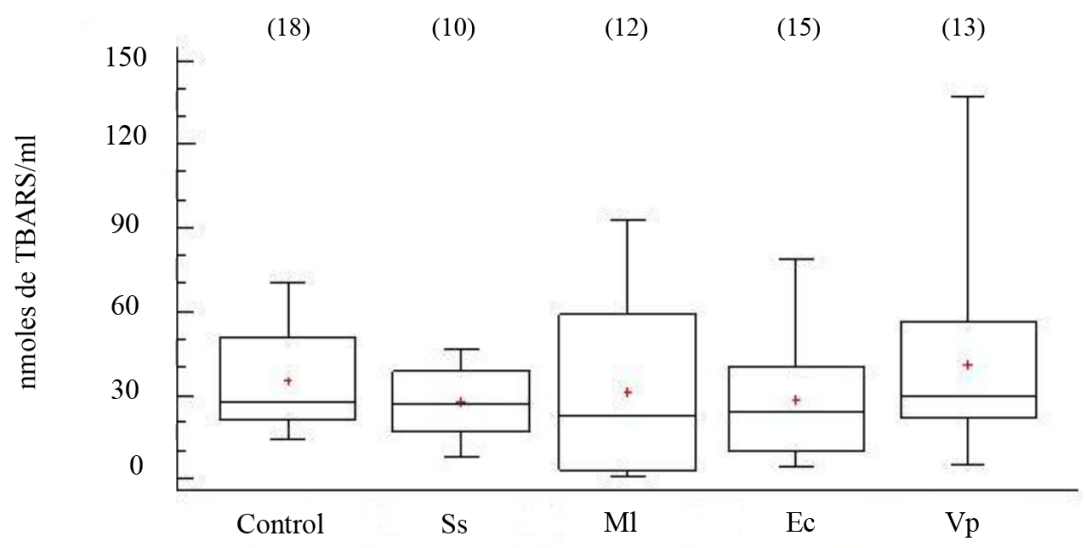

Fig. 6. Concentraciones de sustancias que reaccionan al ácido tiobarbitúrico (TBARS) en fluido celómico del erizo de mar Echinometra lucunter inoculados con solución salina (Ss), M. lysodeikticus (M1), E. coli (Ec) y V.parahaemolyticus $(\mathrm{Vp})$. $(\mathrm{N})$ indica en número de individuos analizados

Fig. 6. Concentrations of thiobarbituric acid reactive substances (TBARS) in coelomic fluid of Echinometra lucunter inoculated with saline solution (Ss), M. lysodeikticus (Ml), E. coli (Ec), and V. parahaemolyticus (Vp). (N) Number of individuals analyzed 


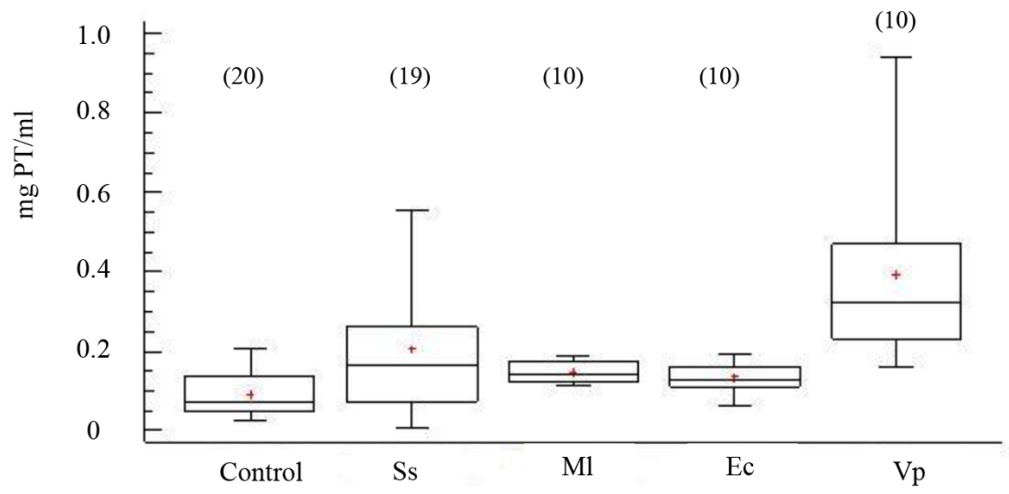

Fig. 7. Concentración de proteínas (PT) en fluido celómico del erizo de mar Echinometra lucunter inoculados con solución salina (Ss), M. lysodeikticus (Ml), E. coli $(\mathrm{Ec})$ y $V$. parahaemolyticus $(\mathrm{Vp})$. (*) El asterisco muestra diferencias significativas $* P<0.05$. (N) indica en número de individuos analizados

Fig. 7. Protein concentrations in coelomic fluid of Echinometra lucunter inoculated with saline solution (Ss), M. lysodeikticus (Ml), E. coli (Ec), and V. parahaemolyticus $(\mathrm{Vp})$. $(*)$ The asterisk shows significant differences $* P<0.05$. (N) Number of individuals analyzed

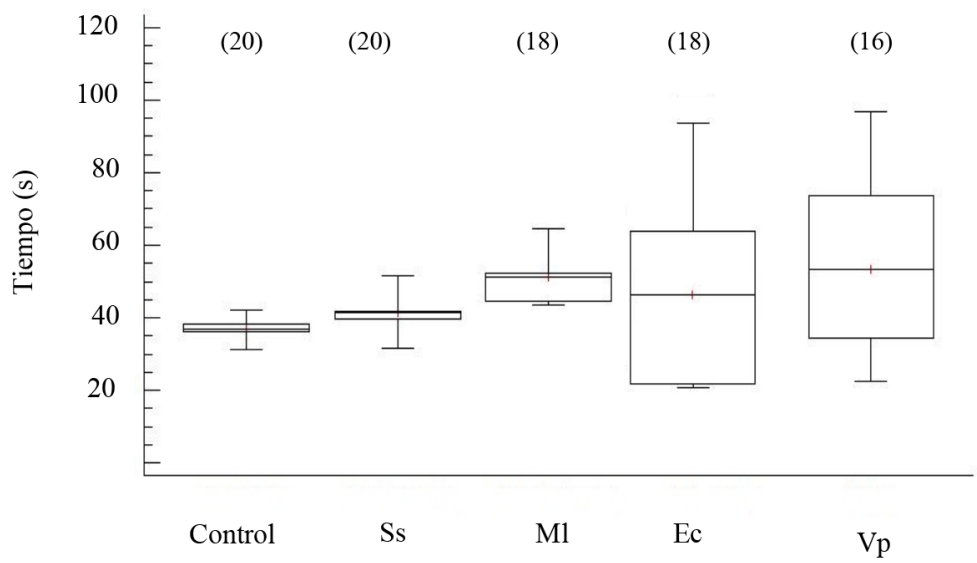

Fig. 8. Tiempo de recuperación invertida en el erizo de mar Echinometra lucunter inoculados con solución salina $(\mathrm{Ss})$, M. lysodeikticus $(\mathrm{Ml})$, E. coli $(\mathrm{Ec})$ y $V$. parahaemolyticus $(\mathrm{Vp})$. $(\mathrm{N})$ indica en número de individuos analizados Fig. 8. Time of righting response in Echinometra lucunter inoculated with saline solution (Ss), M. lysodeikticus (Ml), E. coli (Ec). and V. parahaemolyticus (Vp). (N) Number of individuals analyzed 
Entre el 20 al 35\% de los organismos inyectados con M. lysodeikticus, E. coli y V. parahaemolyticus mostraron ligeros dobleces y pérdidas entre 1 al $10 \%$ de las espinas en su testa. Sin embargo, tanto en los organismos no inyectados e inyectados con solución salina y cepas bacterianas no se evidenciaron síntomas de enfermedades, al presentar movimientos uniformes de espinas, coloración característica de la testa (negruzca-morada), estimulación oral, movimiento de los tubos digestivos y coloración del FC con tonos entre rosado claro a oscuro. No fueron observados desoves aparentes en el agua del acuario en los organismos experimentales.

\section{DISCUSIÓN}

El incremento significativo en la actividad fagocítica demuestra el estallido oxidativo en los erizos de mar inoculados con las bacterias M. lysodeikticus, E. coli y V. parahaemolyticus, fundamentado en la activación de los sistemas bioquímicos que resultan en la formación de especies reactivas del oxígeno (ERO). En E. lucunter, el incremento en la formación de anión superóxido $\left({ }^{\bullet} \mathrm{O}_{2}\right)$, asociado a la capacidad de fagocitosis, representan la típica respuesta de estallido respiratorio en los celomocitos, al igual que ocurre en las células blancas de mamíferos. $\mathrm{El} \bullet^{\circ} \mathrm{O}_{2}$ tiene un rol preponderante como sustancia de acción bactericida, detalles que han sido demostrados de manera similar en hemocitos de moluscos-bivalvos (Campa-Cordova et al. 2002; Donaghy et al. 2012).

La formación de las ERO es mediada por la enzima NADPH oxidasa contenida en las membranas de las células fagocitarias (Pipe, 1992; Buggé et al. 2006; Donaghy et al. 2012). En camarones y moluscos se ha demostrado la producción de intermediarios reactivos del oxígeno en fagocitos de organismos estimulados por bacterias (Roch, 1999; Campa-Cordova et al. 2002). En el camarón Litopenaus vannamei se reportaron incrementos en el número de celomocitos circundantes en el FC al ser estimulados con distintos antígenos (durante 24 a 48 horas) (Yeh \& Chen 2008). También se observó un aumento en la actividad fagocítica, acción de fenoloxidasas y estallido respiratorio. Los autores observaron que tal estímulo permitió la resistencia de los organismos contra una cepa de Vibrio alginolyticus. En E. lucunter, los celomocitos (amebocitos) se presentan en un $77 \%$ en el FC, los cuales poseen, junto a otros tipos de células, una alta capacidad de realizar la fagocitosis (Tucunduva-Faria \& Machado-Cunha da Silva, 2008), lo que sugiere su aporte significativo en la formación de las ERO.

La estimulación de las respuestas del sistema inmunológico celular y humoral en erizos de mar, estimulados 
con bacterias vivas, ha sido evidenciada en otras especies de erizos de mar tales como Lytechinus variegatus (Bastidas, 2017), Strongylocentrotus lividus (Dheilly, 2010) y Sterechinus neumayeri (Ovando, 2009). Cuando la fagocitosis es un proceso activo, las ERO pueden sobrepasar la capacidad de las defensas antioxidantes y producir desequilibrios moleculares (Dolmatova et al. 2003; Majeske et al. 2013), especialmente en los lípidos de membranas. Este daño oxidativo molecular dependerá de la acción barredora de las defensas antioxidantes celulares. En nuestro estudio, la elevada actividad fagocítica en E. lucunter conllevó a una activación del sistema antioxidante enzimático, fundamentado con el incremento de la actividad de la CAT. Posiblemente, la formación de $\bullet^{\bullet} \mathrm{O}_{2}$ durante el proceso de fagocitosis y su posterior trasformación a $\mathrm{H}_{2} \mathrm{O}_{2}$ (por la enzima SOD), permiten la sobreproducción de este último, sirviendo de sustrato a la CAT.

CAT puede mantener en concentraciones fisiológicamente estables al $\mathrm{H}_{2} \mathrm{O}_{2}$, descomponiéndolo en $2 \mathrm{H}_{2} \mathrm{O}$ y $\mathrm{O}_{2}$, limitando así, la sobreproducción de radicales libres de alta toxicidad, tal como ${ }^{\circ} \mathrm{OH}$ (Storey, 1996). Beaumont (2010) muestra que los mayores promedios de lípidos peroxidados y daños a proteínas en el erizo de mar Evechinus chloroticus se encuentran asociados a un incremento de la producción de $\mathrm{H}_{2} \mathrm{O}_{2}$. Estos hallazgos fun- damentan la acción antioxidante de CAT para evitar la propagación del daño oxidativo, especialmente en las membranas de los celomocitos de $E$. lucunter, sensibilizados con las cepas de bacterias.

A pesar de la formación intracelular de ${ }^{\bullet} \mathrm{O}_{2}$ en organismos inmuno-estimulados, la actividad de SOD no presentó variación. Posiblemente, la actividad de la SOD es suficiente para eliminar ${ }^{\bullet} \mathrm{O}_{2}$. En crustáceos se ha demostrado la eficiencia de remover el - $\mathrm{O}_{2}$ por parte de la SOD (Holmblad \& Söderhäll, 1999; Campa-Cordova et al. 2002). En otros estudios realizados con el pepino de mar Eupentacia fradatrix, se demostró la producción de ${ }^{\bullet} \mathrm{O}_{2}$, junto a SOD, en amebocitos en presencia tanto de la bacteria Yersinia pseudotuberculosis como de su toxina incubados por 24 horas (Dolmatova et al. 2003).

Las proteínas en el FC de E. lucunter desempeñan un papel dominante en el metabolismo celular y actividad inmune debido a su doble rol, tanto estructural como funcional. El aumento significativo en los niveles de proteínas totales en el FC de los erizos de mar inmuno-estimulados con las distintos microorganismos, en especial a $V \cdot p a-$ rahemolyticus, forma parte de las respuestas inespecíficas inmuno-modulatorias. Parece ser una respuesta común que las proteínas y algunos péptidos son de relativa abundancia cuando los erizos de mar son invadidos por microbios. Adicionalmente, Dheilly (2010) 
observó inducción en las proteínas de estrés y desintoxicadoras en $S$. purpuratus inyectado con lipopolisacáridos (LPS) bacterianos. En el erizo de mar Paracentrotus lividus, sensibilizados con probióticos Lactobacillus paracasei, un recombinante de la misma especie y $S$. cerevisiae, se ha observado, que pueden incrementar las respuestas inmunes tales como la actividad de lisozimas y producción de óxido nítrico (NO). De la misma manera, en P. livi$d u s$ se ha demostrado que la aplicación de zymozán A en organismos infectados con vibrios proveen efectos beneficiosos en la función inmune del organismo (Cipriano-Maack et al. 2016).

Los niveles de tioles totales (-SH) no mostraron variabilidad en el FC de los organismos, aparentemente sanos e inoculados con las distintas cepas bacterianas. Se conoce que el glutatión reducido (GSH) es uno de los componentes antioxidantes de mayor abundancia en las células y el cual contiene un grupo -SH (Dale \& Russo, 1988; Van-Laer et al. 2013). GSH es la principal protagonista en la destrucción de intermediarios de ERO, que cumple una serie de funciones como mantener el balance redox en las células, protegiéndola del estrés oxidativo (Lawrence \& Balzhin, 1998). Este tiol de baja masa molecular actúa como antioxidante endógeno y amortiguador redox, lo que es importante en la homeostasis celular.

\section{CONCLUSIONES}

Los incrementos de la actividad fagocítica, la actividad de CAT y niveles de proteínas en el FC del erizo negro Echinometra lucunter inmuno-estimulado por inoculación bacteriana son el reflejo de los mecanismos de respuesta del organismo ante la invasión de patógenos o cuerpos extraños. Adicionalmente, se demuestra la capacidad de las defensas antioxidantes, en especial CAT, para evitar lipoperoxidación durante el estallido oxidativo. El sistema inmunológico y antioxidante muestran ser respuestas importantes, las cuales pueden garantizan la sobrevivencia de los organismos en presencia de invasiones bacterianas.

\section{BIBLIOGRAFÍA}

Aebi, H. (1984). Catalase in vitro. Meth. Enzimol., 105, 121-126. https://doi. org/10.1016/S0076-6879(84)05016-3

Anton, Y. \& Salazar, R. (2009). El sistema inmune de los invertebrados. Revista Electrónica de Veterinaria, 10, 1-10.

Baba, S. P. \& Bhatnagar, A. (2018). Role of thiols in oxidative stress. Curr. Opin. Toxicol. https://doi.org/10.1016/j.cotox.2018.03.005

Bastidas, M. (2017). Respuestas inmunológicas innatas en el erizo verdi-blanco Lytechinus variegatus (Echinoidea: Toxopneustidae) infectado experimentalmente con bacterias. Trabajo de grado no publicado. Universidad de Oriente, Venezuela. 
Bauer, J. C. \& Young, C. M. (2000). Epidermal lesions and mortality caused by vibriosis in deep-sea Bahamian echinoids: a laboratory study. Dis. Aquat. Org., 39, 193-199. Doi: 10.3354/dao039193.

Beaumont, T. (2010). The free radical theory of ageing: does it apply to Antarctic and temperate sea urchin. Tesis de doctorado no publicada. University of Atago, New Zealand.

Branco, P., Figueiredo D. \& Da Silva J. (2014). Nuevos conocimientos sobre el sistema inmunitario innato de erizo de mar: coelomocytes como biosensores para el estrés ambiental. OA Biología, 2, 2.

Buggé, D. M., Hégaret, H., Wikfors, G. H. \& Allam, B . (2006). Oxidative burst in hard clam (Mercenaria mercenaria) haemocytes. Fish Shell. Immunol., 23, 188-196. https://doi.org/10.1016/j. fsi.2006.10.006.

Campa-Cordova, A., Hernández-Saavedra, N. Y. \& De Pilippis, R. (2002). Generation of superoxide anion and SOD activity in hemocytes and muscle of American white shrimp (Litopenaeus vannamei) as a response to $\beta$-glucan and sulphate polysaccharide. Fish Shell. Inmunol., 12, 353-366. https://doi.org/10.1006/ fsim.2001.0377.

Chihuailaf, R., Contreras, P. \& Wittwer, F. (2002). Patogénesis del estrés oxidativo: Consecuencias y evaluación en salud animal. Veterinaria México, 33, 265-283.

Cipriano-Maack, A. N. (2016). Immunostimulatory effects of different aspects of aquaculture on the host response in the edible sea urchin, Paracentrotus lividus. Tesis de doctorado no publicada, University College Cork, Ireland.

Cooper, E., Raftos, D., Zhang, Z. \& Kelly, K. (1995). Purification and characterisation of tunicate opsonins and cytoki- ne-like proteins. In J. S. Stolen (Ed). Techniques in Fish Immunology-4: Immunology and pathology of aquatic invertebrates (pp. 43-54). Fair Haven, EE. UU.: SOS Publications.

Couter, G., Warnau, M., Jangoux, M. \& Dubois, P. (1999). Reactive oxygen species (ROS) production by amoebocytes of Asterias rubens (Echinodermata). Fish. Shell. Immunol., 12, 187-200. https://doi.org/10.1006/fsim.2001.0366

Dale, B. \& Russo, P. (1988). Sulfhydryl groups are involved in the activation of sea urchin eggs. Gam. Res., 19(2), 161-168. https://doi.org/10.1002/ mrd.1120190206

Dheilly, N. (2010). Proteomic analysis of sea urchin immune responses and characterization of highly variable immune response proteins. Tesis de doctorado no publicada. Sydney, NSW, Australia. Dheilly, N., Haynes, P., Raftos, D. \& Nair S. (2012). Time course proteomic profiling of cellular responses to immunological challenge in the sea urchin, Heliocidaris erythrogramma. Develop. Comp. Immunol., 37, 243-256. https:// doi.org/10.1016/j.dci.2012.03.006

Donaghy, L., Kraffe, E., Le Go1c, N., Lambert, C., Volety, A. K. \& Soudant, P. (2012). Reactive oxygen species in unstimulated hemocytes of the pacific oyster Crassostrea gigas: A mitochondrial involvement. PLoS ONE, 7, e46594. https://doi.org/10.1371/journal.pone.0046594

Dolmatova, L., Elisekina, M., Timchenko, N., kovalera, A., \& Shitkova, O. (2003). Generation of reractive oxygen species in the different fractions of the coclomocytes of holothurian Eupentacia fraundatris in response to the thermostable toxin of Yersenia pseudotuberculosis in vitro. Chinese J Lim. Oceanol., 21(4), 293-304. https://doi. 
org/10.1007/BF02860423

Ellman, G. L. (1959). Tissue sulfhydryl groups. Arch. Biochem. Biophys., 82, 70-77. https://doi.org/10.1016/00039861(59)90090-6

Halliwell, B. \& Gutteridge, J. M. C. (2015). Free Radicals in Biology and Medicine. New York, EE. UU.: Oxford University Press. http://dx.doi.org/10.1093/ acprof:oso/9780198717478.001.0001

Holmblad, T. \& Söderhäll, K. (1999). Cell adhesion molecules and antioxidant enzymes in a crustacean, possible role in immunity. Aquaculture, 172, 111123. https://doi.org/10.1016/S00448486(98)00446-3

Lawrence, J. \& Balzhin, A. 1998. Life-history strategies and the potential of sea urchins for aquaculture. $J$ Shellfish. Res., 17, 1515-1522.

Majeske, A. J., Bayne, C. J. \& Smith, L. C. (2013). Aggregation of sea urchin phagocytes is augmented in vitro by lipopolysaccharide. Publ. Libr. Sci., 8, 61419. https://doi.org/10.1371/journal. pone. 0061419

Matranga, V., Pinsino, A., Celi, M., Natoli, A., Bonaventura, R., Schröder, H. C. \&, Müller, W. E. G. (2005) Monitoring chemical and physical stress using sea urchin immune cells. In V. Matranga (Ed) Echinodermata (pp. 85-110), New York, EE. UU.: Springer. https://doi. org/10.1007/3-540-27683-1_5

Matranga, V., Pinsino, A., Celi, M., Di Bella, G. \& Natoli, A. (2006). Impacts of UV-B radiation on short-term cultures of sea urchin coelomocytes. Mar. Biol., 149, 25-34. https://doi.org/10.1007/ s00227-005-0212-1

Mydlarz, L., Jones, L. \& Drew Harvell, C. (2006). Innate Immunity, environmental drivers, and disease ecology of marine and freshwater invertebrates. $A n$. Rev. Ecol. Evol. System., 37, 251-88. https://doi.org/10.1146/annurev.ecolsys.37.091305.110103

Nebot, C., Moutet, M., Huet, P., J. Yadan \& Chau-diere, J. (1993). Spectrophotometric assay of superoxide dismutase activity based on the activated autoxidation of a tetracyclic catechol. Anal. Biochem., 214, 442-451. https://doi. org/10.1006/abio.1993.1521

Ohkawa, H., Ohishi N. \& Yagi, K. (1978). Assay for lipid peroxides in animal tissues by thiobarbituric acid reaction. Rev. Anal. Biochem., 95(2), 351-358. https:// doi.org/10.1016/0003-2697(79)90738-3

Ovando, P. (2009). Caracterización celular y molecular de la respuesta inmune en el erizo antártico Sterechinus neumayeri. Tesis de grado no publicada. Universidad de Magallanes, Chile.

Pinsino, A. \& Matranga, V. (2015). Sea urchin immune cells as sentinels of environmental stress. Dev. Comp. Immunol., 49, 198-205. http://dx.doi. org/10.1016/j.dci.2014.11.013

Pipe, R. (1992). Generation of reactive oxygen metabolites by the hemocytes of the mussel Mytilus edulis. Dev. Comp. Immunol., 17(1-2), 211-219. https://doi. org/10.1016/0145-305X(92)90012-2

Reyes-Luján, J., Arrieche, D., Lodeiros-Seijo, C., Zapata-Vívenes, E., Barrios, J. \& Salgado, W. (2015). Ciclo gametogénico de Echinometra lucunter (Linnaeus 1758) (Echinometra: Echinoidea) en el Nororiente de Venezuela. Rev. Biol. Trop., 63, 273-283.

Robinson, H. \& Hogden, C. (1940). Relationship to the protein which bears a quantitative production of a stable color conditions necessary for the proteins: a study of the determination of serum concentration. J. Biol. Chem., 35, 707-725.

Roch, P. (1999). Defense mechanisms and disease prevention in farmed marine 
invertebrates. Aquaculture, 172 (12), 125-145. https://doi.org/10.1016/ S0044-8486(98)00439-6

Sokal, R. \& Rohlf, J. (1981). Biometry: The Principles and Practice of Statistics in Biological Reasearch. New York, EE. UU.: WH Freeman.

Storey, K. (1996). Oxidative stress: animal adaptations in nature. Braz. J. Med. Biol. Res., 29(12), 1715-1733.

Taylor, J., Lovera, C., Whaling, P., Buck, K., Pane, E. \& Barry, J. (2014). Physiological effects of environmental acidification in the deep-sea urchin Strongylocentrotus fragilis. Biogeosciences, 11, 1413-1423. https://doi. org/10.5194/bg-11-1413-2014

Tucunduva-Faria, M. \& Machado-Cunha da Silva, J. (2008). Innate immune response in the sea urchin Echinometra lucunter (Echinodermata). J. Invert. Pathol., 98, 58-62. https://doi.org/10.1016/j. jip.2007.10.004

Van-Laer, K., Hamilton, C. \& Messens, J. (2013). Low-Molecular-Weight Thiols in Thiol-Disulfide Exchange. Antiox. Red. Signal., 18(13), 1642-1653. http:// doi.org/10.1089/ars.2012.4964

Wang, Y., Feng, N., Li, Q., Ding, J., Zhan, Y. Y. \& Chang, Y. Q. (2012a). Isolation and characterization of bacteria associated with a syndrome disease of sea urchin Strongylocentrotus intermedius in North China. Aquacult. Res., 44, 1-10. https://doi.org/10.1111/j.13652109.2011.03073.X

Wang, Y., Chang, Y. Q. \& Lawrence J. M. (2012b). Disease in sea urchin. In J. Lawrence, (Ed), Edible Sea Urchins: Biology and Ecology (pp. 179-186). Amsterdam, Netherlands: Elsevier

Yeh, S. T. \& Chen, J. C. (2008). Immunomodulation by carrageenans in the white shrimp Litopenaeus vannamei and its resistance against Vibrio alginolyticus. Aquaculture, 276(1-4), 22-28. https://doi.org/10.1016/j.aquaculture.2008.01.034 